\title{
Increased Risk of Hypertension Associated with Spondyloarthritis Disease Duration: Results from the ASAS-COMOSPA Study
}

\author{
Mohammad H. Derakhshan, Nicola J. Goodson, Jonathan C. Packham, Raj Sengupta, \\ Anna Molto, Helena Marzo-Ortega, and Stefan Siebert, and the BRITSpA \\ and COMOSPA Investigators
}

\begin{abstract}
Objective. Spondyloarthritis (SpA) is associated with a number of cardiovascular (CV) comorbidities We examined the association of SpA disease duration and delay in diagnosis with CV-related conditions.

Methods. Using data from the COMOSPA study, the associations between SpA disease duration and $\mathrm{CV}$-related conditions were evaluated in univariable and multivariable logistic regression models. Each model examined $1 \mathrm{CV}$-related factor as dependent and "SpA disease duration" as a predictor, adjusted for relevant confounders.

Results. Data from 3923 subjects (median SpA disease duration 5.1 yrs, interquartile range 1.3-11.8 yrs) were available for analysis. The main CV-related conditions were hypertension (HTN; 22.4\%), ischemic heart disease (2.6\%), stroke (1.3\%), and diabetes mellitus (5.5\%). HTN was associated with SpA disease duration in both univariable and multivariable analysis, with an OR of 1.129 (95\% CI $1.072-1.189 ; \mathrm{p}<0.001)$ for each 5 -year increase in SpA disease duration. Other factors associated with HTN were age, male sex, current body mass index, ever steroid therapy, and ever synthetic disease-modifying antirheumatic drug therapy, but not nonsteroidal antiinflammatory drugs (NSAID). In subgroup analysis, the strongest association of HTN and disease duration was seen in subjects with the axial-only SpA phenotype (OR 1.202,95\% CI 1.053-1.372) but not in those with peripheral-only SpA (OR 0.902, 95\% CI 0.760-1.070). The other CV conditions were not associated with SpA disease duration.

Conclusion. Duration of SpA disease in the ASAS-COMOSPA cohort is associated with higher odds of HTN, particularly in those with axial disease, but not with other CV-related conditions. The association with HTN does not appear to be related to NSAID exposure. (First Release January 15 2019; J Rheumatol 2019;46:701-9; doi:10.3899/jrheum.180538)
\end{abstract}

Key Indexing Terms:

SPONDYLOARTHRITIS

COMORBIDITY

HYPERTENSION

CARDIOVASCULAR

DISEASE DURATION

From the Institute of Infection, Immunity and Inflammation, University of Glasgow, Glasgow; Academic Rheumatology, Musculoskeletal Biology, Institute of Chronic Disease and Ageing, University of Liverpool, Liverpool; Haywood Rheumatology Centre, Stoke on Trent; Keele University, Keele; Royal National Hospital for Rheumatic Diseases, Bath, UK; Paris Descartes University, Hôpital Cochin, Paris, France; UK National Institute for Health Research (NIHR) Leeds Biomedical Research Centre, Leeds Teaching Hospitals Trust, and Leeds Institute of Rheumatic and Musculoskeletal Medicine (LIRMM), University of Leeds, Leeds, UK.

The COMOSPA study was performed with financial support from AbbVie, Pfizer, and UCB, which provided an unrestricted grant to the Assessment of Spondyloarthritis international Society. Dr. Derakhshan's work is supported by the British Society for Spondyloarthritis (BRITSpA).

M.H. Derakhshan, MD, FRCP, Clinical Epidemiologist, Institute of Infection, Immunity and Inflammation, University of Glasgow;

N.J. Goodson, MRCP, PhD, Senior Lecturer in Rheumatology, Academic Rheumatology, Musculoskeletal Biology, Institute of Chronic Disease and Ageing, University of Liverpool; J.C. Packham, DM, FRCP, Senior Lecturer in Rheumatology, Haywood Rheumatology Centre, and Keele University; R. Sengupta, MBBS, FRCP, Consultant Rheumatologist, Royal National Hospital for Rheumatic Diseases; A. Molto, MD, PhD,

Consultant Rheumatologist, Paris Descartes University, Hôpital Cochin;

\author{
H. Marzo-Ortega, MRCP, PhD, Consultant Rheumatologist, NIHR Leeds \\ Biomedical Research Centre, Leeds Teaching Hospitals Trust and LIRMM, \\ University of Leeds; S. Siebert, PhD, FRCP, Senior Lecturer in \\ Rheumatology, Institute of Infection, Immunity and Inflammation, \\ University of Glasgow. \\ Address correspondence to S. Siebert, Institute of Infection, Immunity and \\ Inflammation, University of Glasgow, Sir Graeme Davis Building, \\ 120 University Place, Glasgow, G12 8TA, UK. \\ E-mail:Stefan.Siebert@glasgow.ac.uk \\ Accepted for publication September 28, 2018.
}

Spondyloarthritis $(\mathrm{SpA})$ encompasses a number of related inflammatory conditions, characterized by considerable overlap in clinical features, reflecting their shared genetic susceptibility and pathophysiology $1,2,3,4,5,6,7$. In common with other chronic inflammatory diseases such as rheumatoid arthritis (RA), SpA is associated with an increased risk of cardiovascular (CV) comorbidity and increased mortality when compared to the general population ${ }^{8,9,10}$. This associ-

Personal non-commercial use only. The Journal of Rheumatology Copyright (c) 2019. All rights reserved. 
ation is incorporated in treatment guidelines, with specific recommendations to monitor and prevent $\mathrm{CV}$ disease $\mathrm{e}^{11,12,13,14,15}$. However, many of these recommendations are adapted from RA, for which this increased risk is better understood $^{15}$. The risk appears to be predominantly related to systemic inflammatory burden, with accumulating evidence for a reduction in $\mathrm{CV}$ events in patients whose RA improves with a range of immunomodulatory treatments $16,17,18,19$. By contrast, the inflammatory burden in SpA is significantly lower than in RA, with many patients with SpA having a normal acute phase. Over the past decade, it has been recognized that, in keeping with their pathophysiological differences, there appear to be differences in the prevalence and type of CV-related comorbidities seen in RA and SpA. For example, the prevalence of major adverse cardiac events (MACE) appears to be lower in patients with ankylosing spondylitis (AS; also termed radiographic axial $\mathrm{SpA}$ ) than in those with $\mathrm{RA}^{20,21}$. In turn, AS is associated with an increased risk of hypertension (HTN), while psoriatic arthritis (PsA) is associated with increased diabetes and metabolic syndrome $22,23,24$. These comorbidities have traditionally been studied in patients with distinct $\mathrm{SpA}$ subgroups, but in clinical practice, the distinction is often less clear and phenotypes often overlap.

The ASAS-COMOSPA study (Assessment of Spondyloarthritis international Society-COMOrbidities in SpondyloArthritis) is a large global study designed to evaluate comorbidities in patients with $\mathrm{SpA}^{25}$. Initial analysis indicated that the most frequent $\mathrm{CV}$-related condition observed in this population is HTN, although there is significant geographic variation in comorbidities ${ }^{25}$. However, it is not clear whether this increased risk relates to the disease itself, its treatments [particularly nonsteroidal antiinflammatory drugs (NSAID) and corticosteroids], or other factors. Although determining causality would require large prospective longterm studies, the ASAS-COMOSPA cohort offers an opportunity to examine some of these associations in more detail. The aim of our current report was to evaluate the association of disease duration and delay in diagnosis with the development of $\mathrm{CV}$-related comorbidities and risk factors in SpA subjects within the ASAS-COMOSPA cohort.

\section{MATERIALS AND METHODS}

Study design. The current report is an analysis of data from the COMOSPA multicenter and international cross-sectional study, with 22 participating countries throughout 5 continents (Africa, Asia, Europe, North and South America) as previously reported ${ }^{25}$. Briefly, consecutive adult patients attending participating centers who met the ASAS classification criteria (either axial or peripheral) for $\mathrm{SpA}$ according to the treating rheumatologist were included $^{26,27}$. All information was obtained at a study visit by the study investigator or research nurse during a face-to-face interview with the participant, combined with review of the medical record. In addition to the study visit date, the date of diagnosis, and where relevant, date(s) of first musculoskeletal symptoms (back pain, peripheral joint symptoms, enthesitis, or dactylitis) were recorded. The following CV-related comorbidities and risk factors were recorded and confirmed in the medical records: ever diagnosis of HTN, ischemic heart disease (IHD), stroke, diabetes mellitus, and dyslipidemia.
As described in the original COMOSPA paper ${ }^{25}$, in each participating country, consecutive adult patients who were able to understand and complete questionnaires were included. The study was conducted according to guidelines for good clinical practice in all countries. Written informed consent was obtained from all subjects before enrollment.

Data analysis. Central tendencies in each group are presented by median and interquartile range (IQR), unless otherwise stated based on results of normality tests conducted for each group of data. Where necessary, the differences between independent groups were examined using Mann-Whitney U test. To study the association between SpA chronology and CV-related conditions, a number of new time variables were created: first, $\mathrm{SpA}$ disease duration was defined as the period between age at diagnosis of SpA and the date of completing the survey. Second, the delay in SpA diagnosis was defined as the time gap between the first musculoskeletal symptoms of $\mathrm{SpA}$ (i.e., the earliest report of back pain, peripheral joint pain, enthesitis, or dactylitis) and the diagnosis of SpA (Supplementary Figure 1, available with the online version of this article).

The association between SpA disease duration (defined in 5-yr blocks) and $\mathrm{CV}$-related conditions was examined using univariable and multivariable binary logistic regression. Each model comprised $1 \mathrm{CV}$-related condition as dependent and SpA disease duration as predictor adjusted for relevant confounders in 2 stages of adjustments. In the first stage (partial adjustment), confounders were age (continuous), sex (reference: females), current body mass index (BMI; continuous), history of smoking (pack-year), alcohol (reference: non-drinker), ever use of NSAID (reference: none), ever use of steroids (reference: none), ever use of synthetic disease-modifying antirheumatic drugs (DMARD; reference: none), ever use of biological DMARD (reference: none), other relevant factors, and interaction terms, if necessary. Thereafter (full adjustment), the model also included the delay in SpA diagnosis (defined in single years because of the shorter duration than SpA disease duration) in addition to all the factors used earlier. The magnitude of the associations is presented using Wald statistics, OR, and relevant $95 \%$ CI. Potential collinearity was tested using correlation matrix, tolerance, and variation inflation factors in a linear regression model. All significance levels were set to a $\mathrm{p}$ value $<0.05$.

Where positive associations were identified, patients were stratified depending on whether their joint involvement was axial, peripheral, or mixed. Axial involvement was defined as the clinician report in the dataset of "ever suffered from inflammatory chronic (at least 3 months) back pain starting before the age of 45 years," while peripheral involvement was defined as the presence of "ever suffered from peripheral joint disease/symptoms suggestive of enthesitis/dactylitis."

\section{RESULTS}

Demographic and disease characteristics. A total of 3923 participants had suitable data for analysis. A small proportion $(1.5 \%)$ were excluded because of age $<18$ years $(n=41)$, missing date of visit $(n=15)$, missing date of birth $(n=4)$, and missing date of birth and date of visit $(n=1)$. The number of participants from each country is shown in Supplementary Table 1, available with the online version of this article.

Baseline demographics showed an age range of 18 to 100 years, with a median (IQR) of 42 (32-53) years; 64.9\% $(\mathrm{n}=2547)$ were male. Female patients were significantly older than males [median (IQR): 44.0 (19.0) vs 41.0 (21.0), $\mathrm{p}<0.001]$. Median BMI was 25.3 (IQR 6.2), more than $33.4 \%$ were overweight (BMI 25-29.9), and $19.5 \%$ were obese (BMI $\geq 30)$. Almost a quarter $(23.0 \%)$ of the patients were current smokers, $23.4 \%$ were ex-smokers, and $53.6 \%$ had never smoked. Regarding alcohol consumption, $47.8 \%$ of patients reported drinking no alcohol, $7.5 \%$ were

Personal non-commercial use only. The Journal of Rheumatology Copyright (C) 2019. All rights reserved 
ex-drinkers, $37.5 \%$ currently drink $<3$ units/day, and only $6.7 \%$ currently drink 3 units or more/day.

The median (IQR) age at SpA diagnosis was 33 years (25.0-43.0) while the median (IQR) age at which the first musculoskeletal symptom(s) of SpA appeared was 29.4 years (21.9-39.9) for the entire group (Figure 1). The estimated median (IQR) SpA disease duration was 5.1 years (1.3-11.8) and the estimated median (IQR) delay in SpA diagnosis was 1.1 years $(0.0-5.9)$.

$C V$-related conditions. The prevalence of the main CV-related conditions assessed were ever diagnosis of HTN in 872 (22.4\%), ever diagnosis of IHD in 102 (2.6\%), ever diagnosis of stroke in $50(1.3 \%)$, ever diagnosis of diabetes in $215(5.5 \%)$, and ever diagnosis of dyslipidemia in 643 $(16.6 \%)$.

Association of SpA disease duration with HTN. An association between the HTN and SpA disease duration was found in both univariable and multivariable models (Table 1), the latter conducted with partial and full adjustment. In the partial adjustment model, the risk was calculated taking into account the possible effects and interactions of all main confounders, but without considering the effect of delay in SpA diagnosis. This model showed a statistically significant association between SpA disease duration and HTN (OR 1.11, 95\% CI 1.06-1.16; Supplementary Table 2, available with the online version of this article). The full adjustment model included a further adjustment for delay in SpA diagnosis (Table 1) and indicated that the odds of having a diagnosis of HTN increased by $13 \%$ per each 5-year increase in the duration of SpA (OR 1.13, 95\% CI 1.07-1.19; p < 0.001; Figure 1).

Confounding variables with significant association with HTN were delay in SpA diagnosis (OR 1.01, 95\% CI $1.00-1.02 ; \mathrm{p}=0.033)$, current age (OR $1.09,95 \% \mathrm{CI}$ $1.08-1.10 ; \mathrm{p}<0.001$ ), male sex (OR 1.44, 95\% CI 1.17-1.77; $\mathrm{p}<0.001$ ), current BMI (OR 1.09, 95\% CI 1.07-1.11; $\mathrm{p}<0.001$ ), ever use of steroids (OR 1.23, 95\% CI 1.01-1.50; $\mathrm{p}=0.038$ ), and ever use of synthetic DMARD (OR 1.34, 95\% CI 1.09-1.66; $\mathrm{p}=0.006$ ), but not ever use of NSAID or biologic DMARD (Table 1).

Association of SpA disease duration with HTN in SpA subgroups. To evaluate whether the association was generalized for SpA or related to joint distribution, participants were stratified into those with axial disease only, peripheral disease only, mixed axial, and peripheral disease, any axial disease (axial only plus mixed), and any peripheral (peripheral disease only plus mixed; Supplementary Figure 2, available with the online version of this article). Stratification by SpA subgroup indicated a stronger association was found in the "axial only" subgroup (OR 1.20,95\% CI $1.05-1.37, \mathrm{p}=0.007)$ compared to "peripheral only" subgroup (OR 0.90, 95\% CI 0.76-1.07, $\mathrm{p}=0.237$; Table 2). Similarly, comparing the "any axial" subgroup to the "any peripheral" subgroup showed slightly higher odds of HTN in the former group. The results in the "mixed" axial and peripheral subgroup were comparable to that of the entire cohort (Table 2).

Analyses of associations with partial adjustments (all confounders excluding delay in SpA diagnosis) produced similar estimates to those for the full adjustment models.

Association of delay in SpA diagnosis with HTN. There was a weak but marginally significant association between HTN and the delay in SpA diagnosis (OR 1.01, 95\% CI 1.00-1.02; $\mathrm{p}=0.033)$ in the entire cohort, indicating a $1 \%$ increase in the odds of HTN for each year of delay in SpA diagnosis (Table 1; Figure 1). Stratifying the results by SpA subgroups revealed that the association was largest in the "any axial" (OR 1.02, 95\% CI 1.01-1.03; $\mathrm{p}=0.004)$ and "mixed axial and peripheral" subgroups (OR 1.02, 95\% CI 1.01-1.03; $\mathrm{p}=0.002$; Table 2).

Associations of SpA disease duration with other CV-related
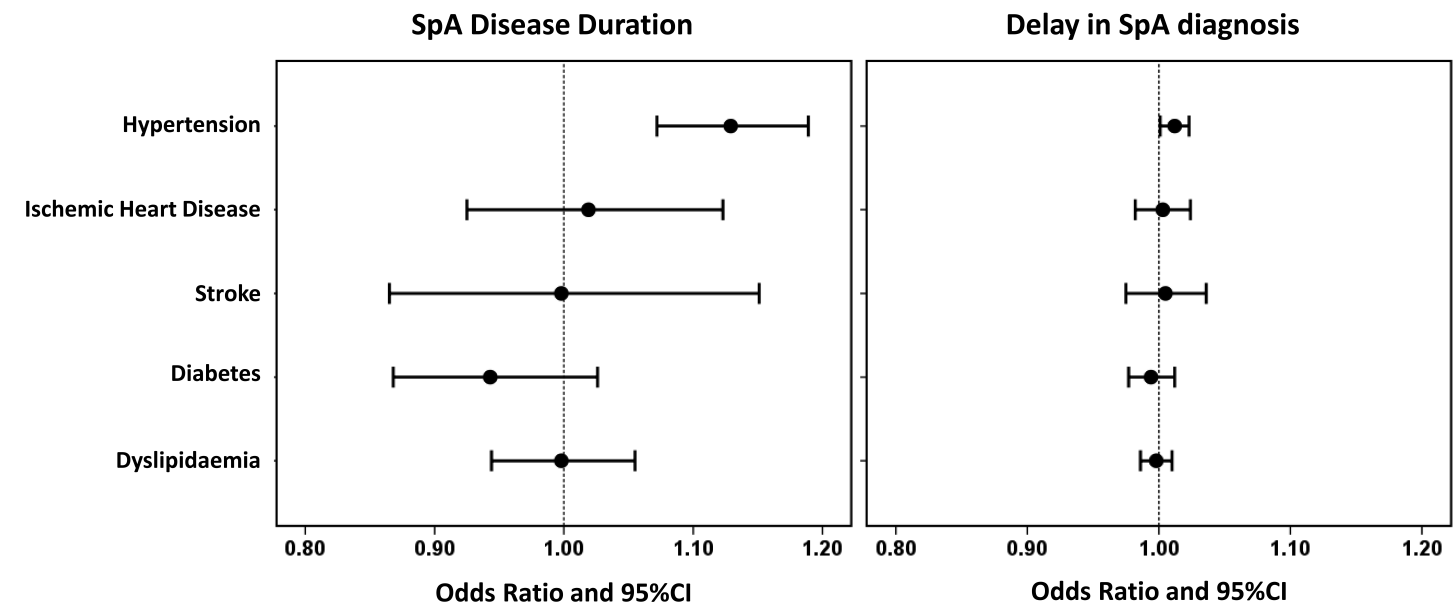

Figure 1. Summary results of logistic regression showing OR (black circles) and 95\% CI (lines) for the associations of SpA disease duration and delay in diagnosis with the various cardiovascular-related conditions. SpA: spondyloarthritis.

Personal non-commercial use only. The Journal of Rheumatology Copyright (C) 2019. All rights reserved. 
Table 1. Association between SpA disease duration and hypertension, adjusted for all relevant confounders including delay in SpA diagnosis; entire cohort.

\begin{tabular}{lcccc}
\hline All & Wald Test & $\mathrm{p}$ & OR & 95\% CI for OR \\
\hline Univariable & & & & \\
$\quad$ SpA disease duration, 5-yr blocks & 253.050 & $<0.001$ & 1.387 & $1.332-1.444$ \\
Multivariable & & & & \\
SpA disease duration, 5-yr blocks & 21.031 & $<0.001$ & 1.129 & $1.072-1.189$ \\
Delay in SpA diagnosis & 4.521 & 0.033 & 1.012 & $1.001-1.023$ \\
Age, yrs & 360.371 & $<0.001$ & 1.089 & $1.080-1.099$ \\
Sex (ref: female) & 12.196 & $<0.001$ & 1.442 & $1.174-1.771$ \\
Current BMI & 108.154 & $<0.001$ & 1.089 & $1.072-1.107$ \\
Smoking, pack-yr & 0.017 & 0.895 & 1.000 & $0.993-1.006$ \\
Alcohol (ref: never) & 5.613 & 0.132 & & \\
$\quad$ Ex-drinker & 0.838 & 0.360 & 1.185 & $0.824-1.706$ \\
$\quad$ Current, $<3$ units/day & 2.848 & 0.092 & 0.835 & $0.678-1.029$ \\
$\quad$ Current, $\geq 3$ units/day & 1.353 & 0.245 & 0.794 & $0.539-1.171$ \\
Ever use of NSAID & 0.514 & 0.473 & 1.125 & $0.816-1.551$ \\
Ever use of steroids & 4.308 & 0.038 & 1.231 & $1.012-1.497$ \\
Ever use of synthetic DMARD & 7.451 & 0.006 & 1.341 & $1.086-1.655$ \\
Ever use of biologic DMARD & 0.244 & 0.622 & 1.050 & $0.866-1.272$ \\
\hline
\end{tabular}

SpA: spondyloarthritis; BMI: body mass index; NSAID: nonsteroidal antiinflammatory drugs; DMARD: disease-modifying antirheumatic drugs.

Table 2. Association between hypertension and SpA disease duration or delay in SpA diagnosis in entire group and subgroups of SpA (multivariable).

\begin{tabular}{|c|c|c|c|c|c|}
\hline & Group N & Wald Test & $\mathrm{p}$ & OR & $95 \% \mathrm{CI}$ \\
\hline \multicolumn{6}{|l|}{ SpA disease duration (5-yr blocks $)^{1}$} \\
\hline Entire group & 3923 & 21.031 & $<0.001$ & 1.129 & $1.072-1.189$ \\
\hline Any peripheral & 2688 & 12.591 & $<0.001$ & 1.109 & $1.047-1.174$ \\
\hline Axial only & 1138 & 7.393 & 0.007 & 1.202 & $1.053-1.372$ \\
\hline Peripheral only & 434 & 1.397 & 0.237 & 0.902 & $0.760-1.070$ \\
\hline Entire group & 3923 & 4.521 & 0.033 & 1.012 & $1.001-1.023$ \\
\hline Any axial & 3393 & 8.397 & 0.004 & 1.017 & $1.006-1.029$ \\
\hline Any peripheral & 2688 & 5.019 & 0.025 & 1.013 & $1.002-1.025$ \\
\hline Axial only & 1138 & 0.321 & 0.571 & 1.008 & $0.980-1.038$ \\
\hline Peripheral only & 434 & 1.026 & 0.311 & 0.978 & $0.936-1.021$ \\
\hline Mixed axial and peripheral & 2254 & 9.420 & 0.002 & 1.020 & $1.007-1.033$ \\
\hline
\end{tabular}

\footnotetext{
${ }^{1}$ The model adjusted to potential confounders including age, sex, BMI, smoking, alcohol consumption, ever use of NSAID, ever use of steroids, ever use of synthetic DMARD, ever use of biologic DMARD, and delay in SpA diagnosis. ${ }^{2}$ Adjusted to all above factors, including SpA disease duration but not delay in SpA diagnosis. SpA: spondyloarthritis; BMI: body mass index; NSAID: nonsteroidal antiinflammatory drugs; DMARD: disease-modifying antirheumatic drugs.
}

conditions. Other CV-related conditions including IHD, stroke, diabetes mellitus (Tables 3-5, and summarized in Figure 1), and dyslipidemia (Supplementary Table 3, available with the online version of this article) were incorporated into logistic regression models, adjusting for relevant confounders with no associations found with SpA disease duration, in either univariable or multivariable models. Similarly, delay in SpA diagnosis was not associated with any of these conditions.

\section{DISCUSSION}

The large COMOSPA cohort has allowed for the evaluation of the association of a number of CV-related conditions with disease duration across the spectrum of SpA. In this analysis, we found that HTN was associated with increased SpA disease duration and delay in SpA diagnosis, even when adjusted for other confounding factors. This association was stronger in patients with axial disease than in those with peripheral disease. There was no association of SpA disease duration with IHD, stroke, diabetes mellitus, or dyslipidemia.

The association of CV comorbidities with inflammatory rheumatic conditions is well recognized and widely quoted $^{11,12,13,14,15}$. However, most of the data come from RA, with fewer, smaller studies with significant heterogeneity in 
Table 3. Association between SpA disease duration and ischemic heart disease, adjusted for all relevant confounders including delay in SpA diagnosis; entire cohort.

\begin{tabular}{lcccc}
\hline All & Wald Test & $\mathrm{p}$ & OR & $95 \%$ CI \\
\hline Univariable & & & & \\
$\quad$ SpA disease duration, 5-yr blocks & 55.447 & $<0.001$ & 1.333 & $1.236-1.438$ \\
Multivariable & & & & \\
SpA disease duration, 5-yr blocks & 0.142 & 0.706 & 1.019 & $0.925-1.123$ \\
Delay in SpA diagnosis & 0.059 & 0.808 & 1.003 & $0.982-1.024$ \\
Age, yrs & 50.129 & $<0.001$ & 1.079 & $1.056-1.101$ \\
Sex (ref: female) & 6.710 & 0.010 & 2.100 & $1.198-3.681$ \\
Current BMI & 1.430 & 0.232 & 1.023 & $0.986-1.062$ \\
Smoking, pack-yr & 4.540 & 0.033 & 1.013 & $1.001-1.024$ \\
Alcohol (ref: never) & 1.201 & 0.753 & & \\
$\quad$ Ex-drinker & 0.104 & 0.747 & 1.140 & $0.515-2.524$ \\
$\quad$ Current, <3 units/day & 0.478 & 0.489 & 0.835 & $0.500-1.393$ \\
$\quad$ Current, $\geq 3$ units/day & 0.546 & 0.460 & 0.713 & $0.291-1.747$ \\
Ever use of NSAID & 0.450 & 0.502 & 1.301 & $0.603-2.807$ \\
Ever use of steroids & 1.597 & 0.206 & 1.353 & $0.847-2.161$ \\
Ever use of synthetic DMARD & 2.675 & 0.102 & 1.564 & $0.915-2.673$ \\
Ever use of biologic DMARD & 0.108 & 0.742 & 1.081 & $0.679-1.722$ \\
Ever diagnosis of HTN & 25.855 & $<0.001$ & 4.436 & $2.498-7.877$ \\
Ever diagnosis of diabetes & 13.868 & $<0.001$ & 2.774 & $1.621-4.745$ \\
\hline
\end{tabular}

SpA: spondyloarthritis; BMI: body mass index; NSAID: nonsteroidal antiinflammatory drugs; DMARD: disease-modifying antirheumatic drugs; HTN: hypertension.

Table 4. Association between SpA disease duration and stroke, adjusted for all relevant confounders including delay in SpA diagnosis; entire cohort.

\begin{tabular}{lcccc}
\hline All & Wald Test & $\mathrm{p}$ & OR & 95\% CI \\
\hline Univariable & & & & \\
$\quad$ SpA disease duration, 5-yr blocks & 20.107 & $<0.001$ & 1.287 & $1.153-1.437$ \\
Multivariable & & & & \\
$\quad$ SpA disease duration, 5-yr blocks & 0.001 & 0.979 & 0.998 & $0.865-1.151$ \\
Delay in SpA diagnosis & 0.102 & 0.749 & 1.005 & $0.975-1.036$ \\
Age, yrs & 6.969 & 0.008 & 1.042 & $1.011-1.074$ \\
Sex (ref: female) & 0.004 & 0.952 & 0.978 & $0.472-2.025$ \\
Current BMI & 0.495 & 0.482 & 0.980 & $0.925-1.037$ \\
Smoking, pack-yr & 5.121 & 0.024 & 1.017 & $1.002-1.033$ \\
Alcohol (ref: never) & 1.405 & 0.704 & & \\
$\quad$ Ex-drinker & 0.184 & 0.668 & 0.750 & $0.201-2.796$ \\
$\quad$ Current, <3 units/day & 0.268 & 0.605 & 1.202 & $0.598-2.417$ \\
$\quad$ Current, $\geq 3$ units/day & 0.551 & 0.458 & 0.561 & $0.122-2.578$ \\
Ever use of NSAID & 0.126 & 0.723 & 1.219 & $0.408-3.644$ \\
Ever use of steroids & 0.396 & 0.529 & 0.804 & $0.408-1.586$ \\
Ever use of synthetic DMARD & 2.222 & 0.136 & 0.593 & $0.298-1.179$ \\
Ever use of biologic DMARD & 0.019 & 0.891 & 1.046 & $0.546-2.006$ \\
Ever diagnosis of HTN & 21.433 & $<0.001$ & 8.843 & $3.514-22.252$ \\
Ever diagnosis of diabetes & 2.173 & 0.140 & 1.843 & $0.817-4.156$ \\
FHx of MI & 6.436 & 0.011 & 0.423 & $0.217-0.822$ \\
\hline
\end{tabular}

SpA: spondyloarthritis; BMI: body mass index; NSAID: nonsteroidal antiinflammatory drugs; DMARD: disease-modifying antirheumatic drugs; HTN: hypertension; FHx: family history; MI: myocardial infarction.

results in $\mathrm{SpA}^{20,28}$. Several reports have suggested that, when appropriately adjusted for age and sex, the risk of MACE in PsA and AS may be less than in $\mathrm{RA}^{20,29,30}$. In fact, in several AS studies, the lower limit of the $95 \%$ CI for the odds of IHD was 1.0 or less ${ }^{20,21,31}$, whereas others report IHD rates similar to $\mathrm{RA}^{32,33,34}$. The prospective CARdiovascular in rheuMAtology (CARMA) project reported higher prevalence of CV disease in RA than in AS and PsA, with disease duration associated with increased risk of $\mathrm{CV}$ risk factors in all groups $^{35}$. 
Table 5. Association between SpA disease duration and diabetes, adjusted for all relevant confounders including delay in SpA diagnosis; entire cohort.

\begin{tabular}{lcccc}
\hline All & Wald Test & $\mathrm{p}$ & OR & $95 \%$ CI \\
\hline Univariable & & & & \\
$\quad$ SpA disease duration, 5-yr blocks & 27.441 & $<0.001$ & 1.184 & $1.112-1.262$ \\
Multivariable & & & & \\
$\quad$ SpA disease duration, 5-yr blocks & 1.867 & 0.172 & 0.943 & $0.868-1.026$ \\
Delay in SpA diagnosis & 0.440 & 0.507 & 0.994 & $0.977-1.012$ \\
Age, yrs & 41.189 & $<0.001$ & 1.050 & $1.035-1.066$ \\
Sex (ref: female) & 5.254 & 0.022 & 1.543 & $1.065-2.235$ \\
Current BMI & 63.528 & $<0.001$ & 1.095 & $1.071-1.120$ \\
Smoking, pack-yr & 0.095 & 0.757 & 1.002 & $0.991-1.012$ \\
Alcohol (ref: never) & 14.507 & 0.002 & & \\
$\quad$ Ex-drinker & 0.109 & 0.742 & 1.100 & $0.625-1.935$ \\
$\quad$ Current, <3 units/day & 12.746 & $<0.001$ & 0.492 & $0.333-0.726$ \\
$\quad$ Current, $\geq 3$ units/day & 0.729 & 0.393 & 0.750 & $0.388-1.450$ \\
Ever use of NSAID & 0.170 & 0.680 & 0.897 & $0.534-1.506$ \\
Ever use of steroid & 0.369 & 0.544 & 1.111 & $0.791-1.561$ \\
Ever use of synthetic DMARD & 0.521 & 0.471 & 1.149 & $0.788-1.677$ \\
Ever use of biologic DMARD & 1.862 & 0.172 & 1.263 & $0.903-1.768$ \\
Ever diagnosis of HTN & 52.893 & $<0.001$ & 4.067 & $2.787-5.936$ \\
Ever diagnosis of stroke & 1.944 & 0.163 & 1.757 & $0.796-3.881$ \\
FHx of MI & 3.712 & 0.054 & 0.695 & $0.480-1.006$ \\
\hline
\end{tabular}

SpA: spondyloarthritis; BMI: body mass index; NSAID: nonsteroidal antiinflammatory drugs; DMARD: disease-modifying antirheumatic drugs; HTN: hypertension; FHx: family history; MI: myocardial infarction.

The differences in reported rates of MACE associated with PsA and AS relate to several factors, including cohort size, the definition of disease used, and adjustment for confounding factors. Age and sex are particularly important because patients with AS are more likely to be male (therefore already at increased risk for CV disease) and to develop disease at a younger age, and so will have had a longer duration of disease by a specified age than patients with RA. Almost two-thirds (65\%) of the COMOSPA participants were male, with a median age at SpA diagnosis of 33 years and estimated median SpA disease duration of 5.1 years.

Cross-sectional studies can only indicate association and do not imply causality. Attempts have been made to evaluate a possible dose-response relationship between the rheumatic inflammatory burden and the risk of $\mathrm{CV}$ disease. Although there is some evidence to suggest this may also be the case in $\mathrm{SpA}^{36,37,38}$, this association is difficult to assess in $\mathrm{SpA}$ because many of the disease activity measures [e.g., Bath Ankylosing Spondylitis Disease Activity Index (BASDAI)] are subjective, and acute-phase reactants often do not reflect disease activity.

CV-related conditions generally take many years or decades to develop and become evident, so the duration of the underlying $\mathrm{SpA}$ disease offers an alternative way to further evaluate the association of these conditions. The duration of the SpA disease represents the cumulative amount of time the patient has had the disease and any therapies for this, and therefore represents a surrogate of the SpA disease burden.
The delay from symptom onset to SpA diagnosis represents the period without diagnosis or specific therapy. In our study, we found that neither the length of SpA disease duration nor the delay in diagnosis were associated with development of IHD, stroke, diabetes mellitus, or dyslipidemia.

In contrast, we found that the longer the SpA disease duration, the higher the odds of developing HTN (OR 1.13 for every 5 yrs of SpA disease duration). Further, delay in SpA diagnosis was associated with a small increase in HTN (OR 1.01 for every year of delay in SpA diagnosis; 5\% increased risk for every 5 yrs of delay). Previous publications have suggested that SpA conditions, particularly AS, are associated with an increased risk of $\operatorname{HTN}^{20,21,39,40,41}$. The mechanism for this association is currently unclear. NSAID are known to increase blood pressure ${ }^{42,43}$ and remain the mainstay of treatment in axial SpA $(\operatorname{axSpA})^{13}$, while patients with PsA have an increased risk of metabolic syndrome ${ }^{22,23,24}$. Interestingly, NSAID were not associated with HTN in this cohort. This may be because almost $90 \%$ of participants received NSAID at some stage for their symptoms, with no adjustment possible for cumulative dose. Two-thirds (67.8\%) of participants had taken NSAID within the 3 months prior to the study assessment. It is also likely that in clinical practice, NSAID were avoided or stopped in patients with existing HTN.

The association of increased odds of HTN with SpA disease duration was strongest in those patients with axial disease and remained significant, even when corrected for age, sex, and other confounding factors. Because people with

Personal non-commercial use only. The Journal of Rheumatology Copyright @ 2019. All rights reserved. 
AS have longer disease duration, are more likely to be male, and have been shown to be more likely to have subclinical atherosclerosis than those with nonradiographic axSpA ${ }^{44}$, it is possible that the association with HTN would be even more evident in the AS subgroup. However, the available data in this study do not allow us to confidently distinguish AS from nonradiographic axSpA.

Our data therefore suggest that longer SpA disease duration is associated with increased odds of developing HTN, but not IHD or stroke. It should be noted that the crosssectional nature of this study in a selected group of patients with SpA means that no comment can be made regarding the overall prevalence in comparison to the general population. The reasons for the observed differences in HTN and the other CV-related comorbidities in the literature and our study are unclear. It is possible that older age and longer disease duration may have unveiled other CV-related associations. However, from the available literature, it does appear that the prevalence of MACE is higher in RA than in AS and $\mathrm{PsA}^{20,29,30}$, suggesting that $\mathrm{SpA}$ conditions may have less effect on MACE, possibly owing to the lower inflammatory burden. In contrast, metabolic syndrome, which includes HTN, appears to be higher in PsA $22,23,24$. The reason for the increased association of HTN with AS, reported in the literature $^{20,21,41}$ and observed with prolonged disease duration in our study, remains unexplained. While our study did not indicate this was related to NSAID use, these agents are a mainstay of treatment for this condition, and for a long time were the only effective therapy, so it is still possible there is an effect of prolonged use of high-dose NSAID that cannot be detected in our study owing to the limitations related to the NSAID data collection (highlighted elsewhere). This is worthy of further study, including whether the association with HTN is altered by reduced use of NSAID since the introduction of biologic therapies.

The study results must be interpreted in the context of the study limitations. COMOSPA is a cross-sectional study and included self-reported data, which may incur recall bias. However, the clinical staff corroborated the information using the participants' medical records. The prevalence of HTN in this paper $(22.4 \%)$ cannot be compared to the prevalence reported in the earlier COMOSPA paper (33.5\%) because a different definition of HTN was used ${ }^{27}$. We defined HTN as "ever diagnosis of HTN," because we wanted to include only participants with confirmed HTN, whereas the previous paper used a broader definition, which included the current blood pressure reading and history of any antihypertensive medication ${ }^{27}$. Participants with undiagnosed HTN would not be identified in our analysis, but because of the crosssectional nature of the study with a single study timepoint, we did not wish to incorrectly attribute a diagnosis of HTN on the basis of a single blood pressure reading. Similarly, for diabetes mellitus and dyslipidemia, we also used only "ever diagnosis of" and not the current glucose or lipid levels, respectively, because these were not fasting, and these data fields had significant missing data, although sensitivity analysis did not reveal any significant difference (data not shown). The study format did not record the date of onset of HTN, so it is not possible to comment on the timing relative to the SpA disease course. The study design did not rigorously determine cumulative dose or duration of NSAID, so this could not be assessed in the analysis and we were limited to using "ever use of NSAID." We were also unable to analyze the effect of cumulative dose or duration of synthetic or biologic DMARD for the same reasons. There were no measures of physical activity available in the study, so we were not able to incorporate this into our analysis. Patients with IHD or stroke may have been unable to attend clinics or have died, so would not be identified in study selection, leading to underestimation of these comorbidities. While the broad definition of SpA, large numbers, and global distribution in COMOSPA are strengths, they result in significant heterogeneity, and there are likely to be regional variations in diagnostic definitions and management. The broad definition of SpA used in our study also means that no single measure of disease activity is applicable to the entire cohort (e.g., BASDAI not applicable to those with only peripheral disease), so we were unable to examine the effect of current (or previous) disease activity on these comorbidities. The participants were recruited from secondary care clinics, and so may not be representative of the wider population of patients with SpA. The effect of this is likely to vary among countries, with global variation in comorbidities evaluated in other COMOSPA-related analyses. Prospective and longitudinal studies, ideally with record linkage, are required to investigate the observed associations of this and other crosssectional studies.

Our study suggests increased odds of HTN with longer SpA disease duration, particularly in patients with axial disease. Interestingly, disease duration was not associated with the other CV-related conditions in this cohort, adding further support to the growing literature suggesting there are differences in CV-related comorbidities between RA and SpA. Blood pressure measurement is a simple, feasible procedure that should be regularly checked in patients with SpA in the clinical setting, particularly in those with longer disease duration.

\section{REFERENCES}

1. Reveille JD. Genetics of spondyloarthritis - beyond the MHC. Nat Rev Rheumatol 2012;8:296-304.

2. Kirkham BW, Kavanaugh A, Reich K. Interleukin-17A: a unique pathway in immune-mediated diseases: psoriasis, psoriatic arthritis and rheumatoid arthritis. Immunology 2014;141:133-42.

3. Barnas JL, Ritchlin CT. Etiology and pathogenesis of psoriatic arthritis. Rheum Dis Clin North Am 2015;41:643-63.

4. McInnes IB, Schett $G$. The pathogenesis of rheumatoid arthritis. N Engl J Med 2011;365:2205-19.

5. Coates LC, FitzGerald O, Helliwell PS, Paul C. Psoriasis, psoriatic

Personal non-commercial use only. The Journal of Rheumatology Copyright @ 2019 . All rights reserved. 
arthritis, and rheumatoid arthritis: Is all inflammation the same? Semin Arthritis Rheum 2016;46:291-304.

6. Ranganathan V, Gracey E, Brown MA, Inman RD, Haroon N. Pathogenesis of ankylosing spondylitis - recent advances and future directions. Nat Rev Rheumatol 2017;13:359-67.

7. O'Rielly DD, Rahman P. Genetics of psoriatic arthritis. Best Pract Res Clin Rheumatol 2014;28:673-85.

8. Wibetoe G, Ikdahl E, Rollefstad S, Olsen IC, Bergsmark K, Kvien TK, et al. Cardiovascular disease risk profiles in inflammatory joint disease entities. Arthritis Res Ther 2017;19:153.

9. Bengtsson K, Forsblad-d'Elia H, Lie E, Klingberg E, Dehlin M, Exarchou S, et al. Are ankylosing spondylitis, psoriatic arthritis and undifferentiated spondyloarthritis associated with an increased risk of cardiovascular events? A prospective nationwide population-based cohort study. Arthritis Res Ther 2017;19:102.

10. Horreau C, Pouplard C, Brenaut E, Barnetche T, Misery L, Cribier B, et al. Cardiovascular morbidity and mortality in psoriasis and psoriatic arthritis: a systematic literature review. J Eur Acad Dermatol Venereol 2013;27 Suppl 3:12-29.

11. Smolen JS, Landewé R, Bijlsma J, Burmester G, Chatzidionysiou K, Dougados M, et al. EULAR recommendations for the management of rheumatoid arthritis with synthetic and biological disease-modifying antirheumatic drugs: 2016 update. Ann Rheum Dis 2017;76:960-77.

12. Gossec L, Smolen JS, Ramiro S, de Wit M, Cutolo M, Dougados M, et al. European League Against Rheumatism (EULAR) recommendations for the management of psoriatic arthritis with pharmacological therapies: 2015 update. Ann Rheum Dis 2016;75:499-510.

13. van der Heijde D, Ramiro S, Landewé R, Baraliakos X, Van den Bosch F, Sepriano A, et al. 2016 update of the ASAS-EULAR management recommendations for axial spondyloarthritis. Ann Rheum Dis 2017;76:978-91.

14. Coates LC, Kavanaugh A, Mease PJ, Soriano ER, Laura Acosta-Felquer M, Armstrong AW, et al. Group for Research and Assessment of Psoriasis and Psoriatic Arthritis 2015 treatment recommendations for psoriatic arthritis. Arthritis Rheumatol 2016;68:1060-71.

15. Agca R, Heslinga SC, Rollefstad S, Heslinga M, McInnes IB, Peters $\mathrm{MJ}$, et al. EULAR recommendations for cardiovascular disease risk management in patients with rheumatoid arthritis and other forms of inflammatory joint disorders: 2015/2016 update. Ann Rheum Dis 2017;76:17-28.

16. Roubille C, Richer V, Starnino T, McCourt C, McFarlane A, Fleming $\mathrm{P}$, et al. The effects of tumour necrosis factor inhibitors, methotrexate, non-steroidal anti-inflammatory drugs and corticosteroids on cardiovascular events in rheumatoid arthritis, psoriasis and psoriatic arthritis: a systematic review and meta-analysis. Ann Rheum Dis 2015;74:480-9.

17. Solomon DH, Reed GW, Kremer JM, Curtis JR, Farkouh ME, Harrold LR, et al. Disease activity in rheumatoid arthritis and the risk of cardiovascular events. Arthritis Rheumatol 2015; 67:1449-55.

18. Low AS, Symmons DP, Lunt M, Mercer LK, Gale CP, Watson KD, et al. Relationship between exposure to tumour necrosis factor inhibitor therapy and incidence and severity of myocardial infarction in patients with rheumatoid arthritis. Ann Rheum Dis 2017;76:654-60

19. Arida A, Protogerou AD, Konstantonis G, Fragiadaki K, Kitas GD, Sfikakis PP. Atherosclerosis is not accelerated in rheumatoid arthritis of low activity or remission, regardless of antirheumatic treatment modalities. Rheumatology 2017;56:934-9.

20. Eriksson JK, Jacobsson L, Bengtsson K, Askling J. Is ankylosing spondylitis a risk factor for cardiovascular disease, and how do these risks compare with those in rheumatoid arthritis? Ann Rheum Dis 2017;76:364-70.
21. Brophy S, Cooksey R, Atkinson M, Zhou S-M, Husain MJ, Macey $S$, et al. No increased rate of acute myocardial infarction or stroke among patients with ankylosing spondylitis-a retrospective cohort study using routine data. Semin Arthritis Rheum 2012;42:140-5.

22. Dubreuil M, Rho YH, Man A, Zhu Y, Zhang Y, Love TJ, et al. Diabetes incidence in psoriatic arthritis, psoriasis and rheumatoid arthritis: a UK population-based cohort study. Rheumatology 2014;53:346-52.

23. Haroon M, Gallagher P, Heffernan E, FitzGerald O. High prevalence of metabolic syndrome and of insulin resistance in psoriatic arthritis is associated with the severity of underlying disease. J Rheumatol 2014;41:1357-65.

24. Raychaudhuri SK, Chatterjee S, Nguyen C, Kaur M, Jialal I, Raychaudhuri SP. Increased prevalence of the metabolic syndrome in patients with psoriatic arthritis. Metab Syndr Relat Disord 2010;8:331-4.

25. Moltó A, Etcheto A, van der Heijde D, Landewé R, van den Bosch F, Bautista Molano W, et al. Prevalence of comorbidities and evaluation of their screening in spondyloarthritis: results of the international cross-sectional ASAS-COMOSPA study. Ann Rheum Dis 2016;75:1016-23.

26. Rudwaleit M, van der Heijde D, Landewé R, Akkoc N, Brandt J, Chou CT, et al. The Assessment of SpondyloArthritis Internationa Society classification criteria for peripheral spondyloarthritis and for spondyloarthritis in general. Ann Rheum Dis 2011;70:25-31.

27. Rudwaleit M, van der Heijde D, Landewé R, Listing J, Akkoc N, Brandt J, et al. The development of Assessment of SpondyloArthritis international Society classification criteria for axial spondyloarthritis (part II): validation and final selection. Ann Rheum Dis 2009;68:777-83.

28. Polachek A, Touma Z, Anderson M, Eder L. Risk of cardiovascular morbidity in patients with psoriatic arthritis: a meta-analysis of observational studies. Arthritis Care Res 2017;69:67-74.

29. Ogdie A, Yu Y, Haynes K, Love TJ, Maliha S, Jiang Y, et al. Risk of major cardiovascular events in patients with psoriatic arthritis, psoriasis and rheumatoid arthritis: a population-based cohort study. Ann Rheum Dis 2015;74:326-32.

30. Fernández-Gutiérrez B, Perrotti PP, Gisbert JP, Domènech E, Fernández-Nebro A, Cañete JD, et al. Cardiovascular disease in immune-mediated inflammatory diseases: A cross-sectional analysis of 6 cohorts. Medicine 2017;96:e7308.

31. Han C, Robinson DW, Hackett MV, Paramore LC, Fraeman KH, Bala MV. Cardiovascular disease and risk factors in patients with rheumatoid arthritis, psoriatic arthritis, and ankylosing spondylitis J Rheumatol 2006;33:2167-72.

32. Szabo SM, Levy AR, Rao SR, Kirbach SE, Lacaille D, Cifaldi M, et al. Increased risk of cardiovascular and cerebrovascular diseases in individuals with ankylosing spondylitis: a population-based study. Arthritis Rheum 2011;63:3294-304.

33. Jamnitski A, Symmons D, Peters MJ, Sattar N, McInnes I, Nurmohamed MT. Cardiovascular comorbidities in patients with psoriatic arthritis: a systematic review. Ann Rheum Dis 2013;72:211-6.

34. Haroon NN, Paterson JM, Li P, Inman RD, Haroon N. Patients with ankylosing spondylitis have increased cardiovascular and cerebrovascular mortality: a population-based study. Ann Intern Med 2015;163:409-16.

35. Castañeda S, Martín-Martínez MA, González-Juanatey C, Llorca J, García-Yébenes MJ, Pérez-Vicente S, et al; CARMA Project Collaborative Group. Cardiovascular morbidity and associated risk factors in Spanish patients with chronic inflammatory rheumatic diseases attending rheumatology clinics: Baseline data of the CARMA Project. Semin Arthritis Rheum 2015;44:618-26.

36. Berg IJ, Semb AG, van der Heijde D, Kvien TK, Olsen IC, Dagfinrud H, et al. CRP and ASDAS are associated with future 
elevated arterial stiffness, a risk marker of cardiovascular disease, in patients with ankylosing spondylitis: results after 5-year follow-up. Ann Rheum Dis 2015;74:1562-6.

37. Juneblad K, Rantapää-Dahlqvist S, Alenius GM. Disease activity and increased risk of cardiovascular death among patients with psoriatic arthritis. J Rheumatol 2016;43:2155-61.

38. Eder L, Wu Y, Chandran V, Cook R, Gladman DD. Incidence and predictors for cardiovascular events in patients with psoriatic arthritis. Ann Rheum Dis 2016;75:1680-6.

39. Armstrong AW, Harskamp CT, Armstrong EJ. The association between psoriasis and hypertension: a systematic review and meta-analysis of observational studies. J Hypertens 2013;31:433-42.

40. Edson-Heredia E, Zhu B, Lefevre C, Wang M, Barrett A, Bushe CJ, et al. Prevalence and incidence rates of cardiovascular, autoimmune, and other diseases in patients with psoriatic or psoriatic arthritis: a retrospective study using Clinical Practice Research Datalink. J Eur Acad Dermatol Venereol 2015;29:955-63.
41. Ahmed N, Prior JA, Chen Y, Hayward R, Mallen CD, Hider SL. Prevalence of cardiovascular-related comorbidity in ankylosing spondylitis, psoriatic arthritis and psoriasis in primary care: a matched retrospective cohort study. Clin Rheumatol 2016; 35:3069-73.

42. Aljadhey H, Tu W, Hansen RA, Blalock SJ, Brater DC, Murray MD. Comparative effects of non-steroidal anti-inflammatory drugs (NSAIDs) on blood pressure in patients with hypertension. BMC Cardiovasc Disord 2012;12:93.

43. Fournier JP, Sommet A, Bourrel R, Oustric S, Pathak A, Lapeyre-Mestre M, et al. Non-steroidal anti-inflammatory drugs (NSAIDs) and hypertension treatment intensification: a population-based cohort study. Eur J Clin Pharmacol 2012; 68:1533-40.

44. Rueda-Gotor J, Llorca J, Corrales A, et al. Subclinical atherosclerosis is not increased in patients with non-radiographic axial spondyloarthritis. Clin Exp Rheumatol 2016;34:159-60. 